\section{Empirical Relation for the Isothermal Density Derivative of the Optical Dielectric Constant}

For liquids, the Einstein theory ${ }^{1}$ of light scattering, the Gans theory ${ }^{2}$ of the Kerr effect and the Leontovitch theory ${ }^{3}$ of the Maxwell effect all require knowledge of the isothermal density derivative of the optical dielectric constant, $\rho\left(\partial n^{2} / \partial \rho\right)_{T}$. Experimental values of this coefficient have been given for only a few liquids. In the past, resource has been made to dielectric equations of state, such as the Lorentz-Lorenz equation, which relate the dielectric constant to the density, in order to calculate this coefficient. Usually, the dielectric constant of a liquid is not a function of density only, however, but depends separately on pressure $P$ and temperature $T$, so that the coefficients $\left(\partial n^{2} / \partial p\right)_{\varrho}$ and $\left(\partial n^{2} / \partial T\right)_{\varrho}$ are not zero $^{4}$. For example, it has been shown ${ }^{5}$ that the use of the Lorentz-Lorenz equation in the Einstein light scattering equation may lead to errors of between 10 and 20 per cent,

Table 1. DENSITY DIFFERENTIALS

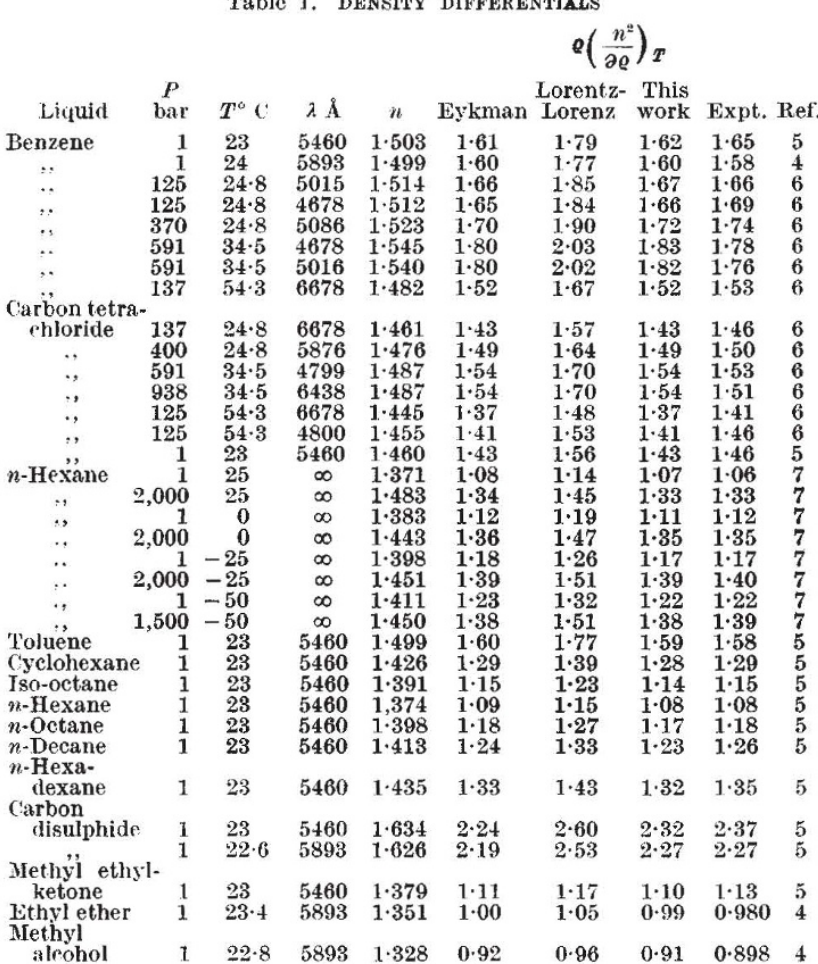

To reduce these errors in the case of liquids for which $\rho\left(\partial n^{2} / \partial \rho\right)_{T}$ has not been measured, the available experi. mental data for common organic liquids have been used to provide an empirical relationship between $\rho\left(\partial n^{2} / \partial \rho\right)_{T}$ and $n$, both quantities being at the same temperature, pressure and wavelength. This relation is

$$
\rho\left(\frac{\partial n^{2}}{\partial \rho}\right)_{T}=\frac{1}{30}\left(n^{2}-1\right)\left(23+7 n^{2}\right)
$$

and fits the experimental data to within about \pm 1 per cent for most liquids for temperatures between $-50^{\circ} \mathrm{C}$ and $+35^{\circ} \mathrm{C}$, pressures from 1 to 1,000 bars and over the whole visible spectrum, although sufficient data to test these extremes of range exist for only a few liquids. Static dielectric constant measurements on $n$-hexane also fit the empirical equation, where the dielectric constant is equated to $n^{2}$. The measurements were made in the $100 \mathrm{kHz}$ region, so the measurement wavelength is effectively infinite. The Lorentz-Lorenz equation

$$
\left(\frac{n^{2}-1}{n^{2}+2}\right)_{p}^{1}=\text { const. }
$$

and the Eykman equation

$$
\left(\frac{n^{2}-1}{n+0 \cdot 4}\right) \frac{1}{p}=\text { const. }
$$

yield density differentials of

$$
\frac{1}{3}\left(n^{2}-1\right)\left(n^{2}+2\right) \text { and } \frac{2 n\left(n^{2-1}\right)(n+0 \cdot 4)}{1+n^{2}+0 \cdot 8 n}
$$

respectively, and these are compared with the proposed empirical formula and experimental values in Table 1. It can be seen that the Lorentz-Lorenz equation gives values of $\rho\left(\partial n^{2} / \partial \rho\right)_{T}$ which are about 8 per cent too high over the whole range, and the Eykman equation is valid only in the region near $n=1 \cdot 5$, being 3.3 per cent low at $n=1 \cdot 6$ and 2 per cent high at $n=1 \cdot 35$. The empirical equation may be integrated to provide a dielectric equation of state st constant temperature, but such an equation of state must necessarily be approximate. It is rather the purpose of this communication to indicate a way of estimating density differentials of the optical dielectric constant to a better accuracy than has hitherto been attained.

Department of Physics,

\section{G. H. Meeten}

Sir John Cass College,

London.

Received March 21, 1968.

${ }^{1}$ Einstein, A., Ann. Physik, 33, 1275 (1910).

${ }^{2}$ Gans, R., Z. Physik, 17, 353 (1923).

${ }^{3}$ Leontovitch, M., J, Phys, USSR, 4, 499 (1941).

4 Raman, V., and Venkataraman, K. S., Proc. Roy. Soc., A, 171, 137 (1939).

${ }^{5}$ Coumou, D. J., Mackor, E. L., and Hijmans, J., Trans. Faraday Soc., 60, 1539 (1964).

' Waxler, R. M., Weir, C. E., and Schamp, H. W., J. Res. Nat. Bur. Stand. 68A, 489 (1964).

${ }^{7}$ Mopsik, F. I., J. Res. Nat. Bur. Stand., 71 A, 287 (1967).

\section{Isotope Shift in Crystal-field Splitting}

MARSHALl et al..$^{1,2}$ have demonstrated experimentally that the isotope shift in the crystal-field splittings of the magnetic ions doped in some host lattices, $\delta D$, is proportional to the mass difference of the isotopic magnetic ions and decreases with temperature. Based on the result that the phonon-induced crystal-field splitting is approximately proportional to the mean square displacement of the atoms ${ }^{3}$, I have shown ${ }^{4}$ that the isotope shift in the crystal-field splittings of two isotopic magnetic ions is approximately proportional to the difference between the mean square displacements of these two isotopes, $\left\langle u_{0}^{2}\right\rangle_{1}$ and $\left\langle u_{0}^{2}\right\rangle_{2}$

$$
\delta D \propto\left(\left\langle u_{0}^{2}\right\rangle_{1}-\left\langle u_{0}^{2}\right\rangle_{2}\right)
$$

In this communication, I shall try to use this result to explain the temperature dependence of the isotope shifts measured by Marshall et al. ${ }^{1}$.

The Einstein model was successfully used by Montgomery to interpret ${ }^{5}$ the difference between the lattice constants of ${ }^{6} \mathrm{Li}$ and ${ }^{7} \mathrm{Li}$. As a result, it could be assumed that the two doped isotopic magnetic ions in a host crystal vibrate with Einstein frequencies $\nu_{1}$ and $\nu_{2}$. These frequencies are related to the masses of the isotopic magnetic ions, $M_{1}^{\prime}$ and $M_{2}^{\prime}$, by the relations $\nu_{i}=b /\left(M_{i}\right)^{1 / 2}, i=1,2$, where $b$ is the proportionality constant related to the force constant. In this case, the mean square displacement is found to be

$$
\left\langle u_{0}^{2}\right\rangle_{i} \propto\left[\frac{1}{2}+\frac{1}{\exp \left(h v_{i} / k T\right)-1}\right], i=1,2
$$

